\title{
The gas temperature compensation research based on TDLAS technology
}

\author{
Chenchen $\mathrm{Hu}^{1, \mathrm{a}^{*}}$, Xiaowei Chen ${ }^{1, \mathrm{~b}}$, Zhengying $\mathrm{Li}^{1,2, \mathrm{c}}$ \\ ${ }^{1}$ National Engineering Laboratory for Fiber Optic Sensing Technology, Wuhan University of \\ Technology, Wuhan, Hubei, China \\ ${ }^{2}$ Key Laboratory of Fiber Optic Sensing Technology and Information Processing, Ministry of \\ Education, Wuhan University of Technology, Wuhan, Hubei, China

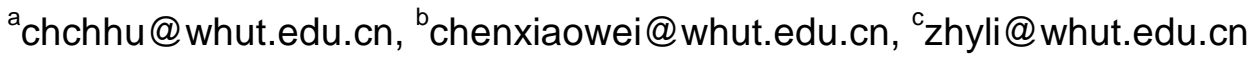

\begin{abstract}
Keywords: Tunable diode laser absorption spectroscopy(TDLAS); Gas concentration detection; Second harmonic; Temperature compensation

Abstract. Tunable diode laser absorption spectroscopy (TDLAS) technology has the characteristics of high sensitivity, selectivity and rapid response. The tunable and narrow linewidth of semiconductor lasers are used in this technique to realize the rapid and accurate online detection of target gas concentration, through the method of selecting the absorption line of the target gas to exclude the interference from other gas. This paper introduces the system and experiment of gas concentration detection based on TDLAS and studies the influence of temperature on gas measurement. Furthermore, a method of temperature measurement to reduce the error of the concentration measurement is presented in this paper, which uses the second harmonic peak ratio of the target line to measure temperature and compensate temperature in the case of unknown concentration. The final experimental results has validated the rationality of the temperature compensation method and further improved the accuracy of the gas concentration detection based on TDLAS technology.
\end{abstract}

\section{Introduction}

With the rapid rising of semiconductor laser industry, the gas spectral absorption detection methods have gradually matured. Among them, the tunable diode laser absorption spectroscopy technology (TDLAS) has become one of the core technology of gas detection. TDLAS has been widely applied in the fields of air pollutants detection, environmental supervision, commercial manufacture and the source of energy and electricity, for its high sensitivity, short response time, non-contact measurement and so $\mathrm{on}^{[1][2]}$. However, due to the dependency between the absorption line type and temperature of the gas, the absorption line intensity and line width will change with the change of the temperature and the concentration of the gas, which has a direct impact on the line type and amplitude of the second harmonic signal and therefore brings about the measurement error. Thus, the temperature measurement is required and the temperature compensation can decrease the measurement error of gas concentration $^{[3][4]}$. Nowadays, the technology of gas temperature measurement mainly contains contact temperature measurement technology and non-contact infrared radiation temperature measurement technology, both of which have demerits when being used. The thermocouple temperature log will probably disturb the temperature field to be examined, causing the measurement error and high temperature and corrosive gas can obviously decrease the life of elements. The infrared radiation measurement cannot disturb the temperature field but can be largely influenced by the gas environment ${ }^{[5][6]}$.

In this paper, the TDLAS system is used to analyze the two absorption spectra, using the second harmonic peak ratio of the target line to measure temperature and the temperature dependence formula of linear intensity in HITRAN data base to compensate temperature in the case of unknown concentration. Thus, the measurement error of the concentration can be reduced and the accuracy of TDLAS sensors for measuring gas concentration can be further improved. 


\section{The principle of gas detection based on TDLAS technology}

We use the tunable diode laser to scan the target gas, when single frequency laser with the frequency of $v$ through the target gas with the temperature of $\mathrm{T}$, the pressure of $\mathrm{P}$, the concentration of $\mathrm{C}$, and the optical distance of L. According to the Beer-Lambert absorption theory, we know that

$$
I(v)=I_{0}(v) \exp [-S(T) \Phi(v) C P L] .
$$

Where, $\mathrm{I}_{0}(\mathrm{v})$ and $\mathrm{I}(\mathrm{v})$ represent the incident light intensity of the laser and emitted light intensity of the laser, linear function determines the shape of the measured gas absorption lines, line strength is a function of its temperature dependence can be expressed as

$$
S(T)=S\left(T_{0}\right) \frac{Q\left(T_{0}\right)}{Q(T)}\left[\frac{T_{0}}{T}\right] \exp \left[-\frac{h c E^{\prime \prime}}{k}\left(\frac{1}{T}-\frac{1}{T_{0}}\right)\right] \times\left[\frac{1-\exp \left(-h c v_{0, i} / k T\right)}{1-\exp \left(-h c v_{0, i} / k T_{0}\right)}\right] .
$$

In the formula, when the reference temperature of $\mathrm{S}\left(\mathrm{T}_{0}\right)$ is $\mathrm{T}_{0}$, we can get the strength of the measured absorption lines from HITRAN spectral database. $Q(T)$ and $Q\left(T_{0}\right)$ is the partition function of the measured gas at different temperatures, $E$ "is the low energy transition state, $v_{0, i}$ is transition frequency, $\mathrm{h}$ is Planck constant, $\mathrm{k}$ is Boltzmann constant, $\mathrm{c}$ is the speed of light. According to Eq.2, we can get the proportion of the strength of the measured absorption lines at $\mathrm{T}$ as follow

$$
R=\frac{S_{1}(T)}{S_{2}(T)}=\frac{S\left[T_{0}, \mathrm{v}_{1}\right]}{S\left[T_{0}, \mathrm{v}_{2}\right]} \exp \left[-\left(\frac{h c}{k}\right)\left(E_{1}^{\prime \prime}-E_{2}^{\prime \prime}\right)\left(\frac{1}{T}-\frac{1}{T_{0}}\right)\right] \text {. }
$$

According to the function, $\mathrm{R}$ is the function of temperature $\mathrm{T}$, we can get the value of $\mathrm{T}$ through measuring the value of $\mathrm{R}$. Thus we choose a pair of acetylene $\left(\mathrm{C}_{2} \mathrm{H}_{2}\right)$ absorption lines with the wave number of $6534.36345 \mathrm{~cm}^{-1}$ (Line A) and $6529.171909 \mathrm{~cm}^{-1}$ (Line B) to measure the temperature. They have a better absorptive capacity and their strong line ratio is the monotonic function of temperature. The big difference of low energy can ensure a higher measurement sensitivity. The frequency interval is small, so they are in the laser frequency tuning rage. The Fig. 1 shows the absorption spectroscopy of the selected line pair at different temperature from $-20^{\circ} \mathrm{C}$ to $60^{\circ} \mathrm{C}$.
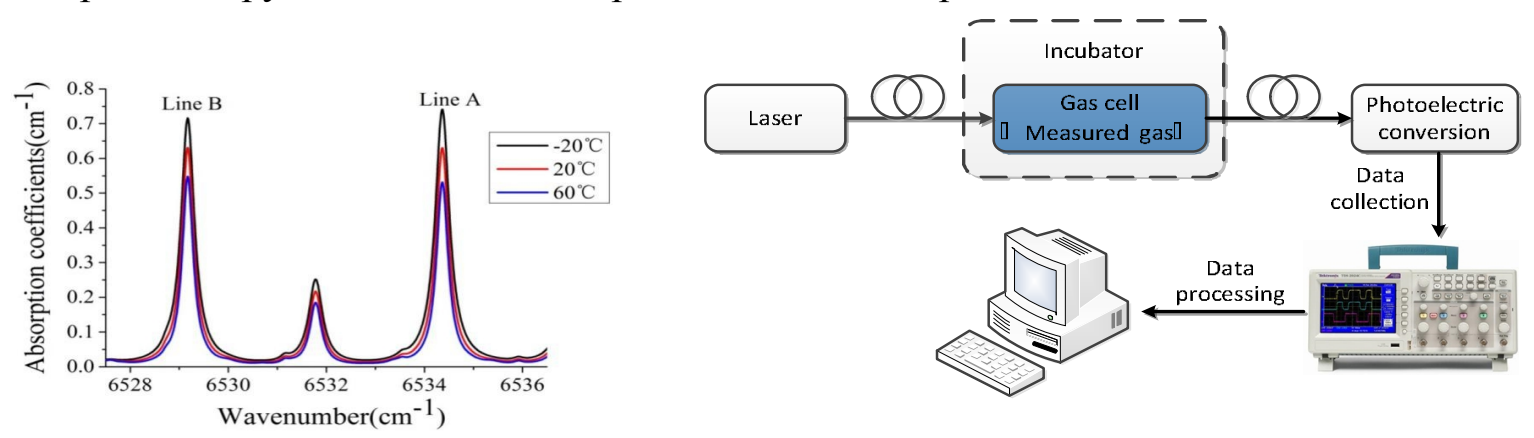

Fig. 1. Absorption spectroscopy of the selected line Fig. 2. Diagram of experiment system structure

In order to restrain a variety of background noise during measurement and improve the detecting resolution, we use wavelength modulation spectroscopy technology. As the absorption of the gas which near the infrared is small, and the concentration of the gas is low in industrial emissions, the second harmonic signal is directly proportional to concentration that

$$
I_{2 f} \propto I_{0} S(T) \Phi(v) C L
$$

Because the gas concentration is unknown, we can't get the temperature through a measurement of the second harmonic signal of a spectral line. So we use a pair of spectral lines to make ration calculation. We can know from Eq.4 that

$$
R_{2 f}=S_{1}(T) I_{0}\left(v_{1}\right) \Phi\left(v_{1}\right) /\left(S_{2}(T) I_{0}\left(v_{2}\right) \Phi\left(v_{2}\right)\right)
$$


According to Eq.5, $\mathrm{R}_{2 \mathrm{f}}$ relates to $\mathrm{R}$ and $\Phi$ after eliminating the influence of the incident light intensity. And $\Phi$ is not only related to T, but also related to the P. But the change of $\mathrm{P}$ almost has the same influence on the linear function of the pair of spectral lines. So, $R_{2 f}$ is mainly decided by $R$, and it is the monotonic function of $\mathrm{T}$. We can realize the measurement of the gas temperature by measuring $\mathrm{R}_{2 \mathrm{f}}$, and we can make temperature compensation through $\mathrm{R}$.

\section{Experimental Device and Process}

The experimental system diagram is shown in Fig. 2, the laser beam from the DFB (Distributed Feedback Laser) pass through the measured gas, and then it is received by sensor through photoelectric conversion to electrical signals. The signals are acquired by the oscilloscope after amplification, filtering and other processing. Then we can get first harmonic signal and the second harmonic signal respectively, after evaluate its peak and thus the concentration of the gas is obtained.

In order to get the second harmonic signal, we use MATLAB to process the signals collected by the oscilloscope. Using peaks correspondence of first harmonic and second harmonic to eliminate the influence of the disturbance of input light intensity on second harmonic peak, then we obtain the peak value of second harmonic in addition to the direct current component as shown in Fig. 3.

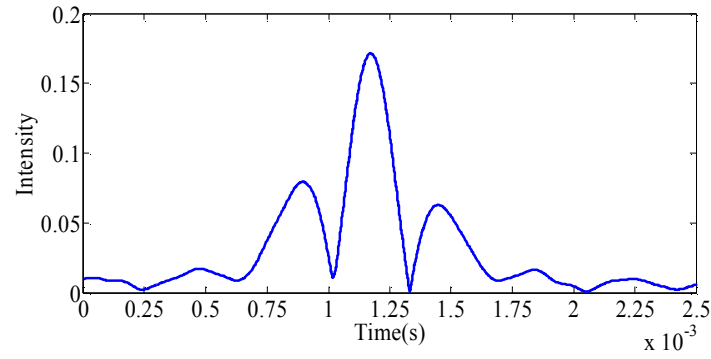

Fig. 3. Waveform of the second harmonic

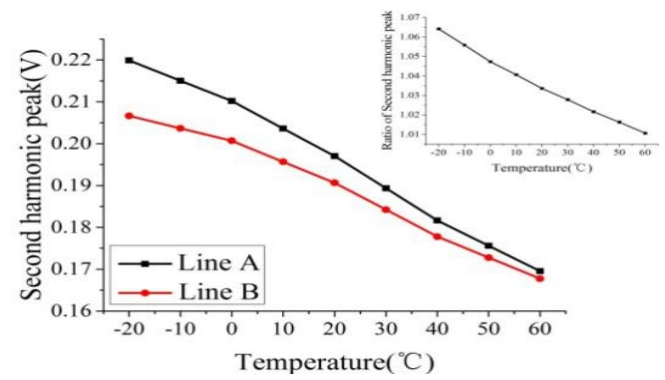

Fig. 4. Second harmonic peak of Line A and Line B

Temperature experiment adopted the $\mathrm{C}_{2} \mathrm{H}_{2}$ gas of $2 \%$, set the temperature from $-20^{\circ} \mathrm{C}$ to $60^{\circ} \mathrm{C}$, record the data oscilloscope acquired, and carry on data processing. As shown in Fig. 4, the second harmonic peak decreases with the increase of temperature. Linear fitting the second harmonic peaks of absorption lines separately, then calculate the ratio to get the curve of the ratio decreases monotonically while the temperature drop as shown in Fig. 4 embedded graphic.

\section{Temperature Compensation}

According to previous analysis, the relationship between the strength of absorption spectral lines and temperature is similar to the variety of the second harmonic peaks when temperature changes. And we can make the measurement of temperature through the ratio of two absorption spectral lines' strength. Thus we can get the value of the line strength of spectral Line A and Line B in HITRAN database. According to it, we can get the curve $\mathrm{S}_{\mathrm{C} 2 \mathrm{H} 2}$ of the value of spectral Line A's strength and the curve of the ratio $\mathrm{R}_{\mathrm{A} / \mathrm{B}}$ of two absorption spectral lines' strength when temperature changes, as shown in Fig. 5. Then we can get the equation of $\mathrm{S}_{\mathrm{C} 2 \mathrm{H} 2}$ and $\mathrm{R}_{\mathrm{A} / \mathrm{B}}$, and record the polynomial parameters in Table 1 .

Combine Eq. 6 with formula Eq.4, set the reference temperature as $\mathrm{T}_{0}$. $\mathrm{C}_{\text {before }}$ is the concentration of $\mathrm{C}_{2} \mathrm{H}_{2}$ before correction, and $\mathrm{C}_{\text {after }}$ is the concentration of $\mathrm{C}_{2} \mathrm{H}_{2}$ after compensation. So we can get the compensation formula shown in Eq.8.

Table 1 Reference value

\begin{tabular}{ccccc}
\hline Equation & Parameter & Value & Parameter Error & $\mathrm{R}^{2}$ \\
\hline $\mathrm{S}_{\mathrm{C} 2 \mathrm{H} 2}$ & $\mathrm{~m}$ & $-4.16667 \mathrm{E}-23$ & $3.63696 \mathrm{E}-25$ & 0.99939 \\
& $\mathrm{n}$ & $1.30778 \mathrm{E}-20$ & $1.18783 \mathrm{E}-23$ & \\
\hline $\mathrm{R}_{\mathrm{A} / \mathrm{B}}$ & $\mathrm{a}$ & $3.42279 \mathrm{E}-6$ & $2.59906 \mathrm{E}-7$ & 0.99964 \\
& $\mathrm{~b}$ & -0.00101 & $1.19481 \mathrm{E}-5$ & \\
& $\mathrm{c}$ & 1.06053 & $2.0443 \mathrm{E}-4$ & \\
\hline
\end{tabular}




$$
\begin{aligned}
& S_{C 2 H 2}(T)=m T+n \\
& R_{A / B}(T)=a T^{2}+b T+c \\
& C_{a f f e r}=C_{b e f o r e}\left(S_{C 2 H 2}\left(T_{0}\right) / S_{C 2 H 2}(T)\right) \\
&
\end{aligned}
$$

Fig. 5. Line strength of Line A and Line B

We can get the ratio of the absorption spectral line of second harmonic peck in the temperature experiment, then put it into Eq. 7 to get the value of measured gas temperature., then use Eq. 8 to make temperature compensation of absorption Line A, which is based on $20^{\circ} \mathrm{C}$. The result shows in Fig. 6 .

According to the result, the second harmonic peak of Line A changes little in different temperature after temperature compensation. And its variation range is only a quarter of that before compensation. Comparing with the standard value in $20^{\circ} \mathrm{C}$, the maximum error of the second harmonic peak of Line A is $5.76 \%$ after compensation, largely reducing the temperature influence on the second harmonic peak of absorption line, improving the accuracy of gas concentration measurement of TDLAS system.

\section{Conclusion}

In this paper, we use TDLAS system to analyze two scanned spectral lines, and get the gas temperature through the ratio of the second harmonic peaks. Then according to temperature relationship equation of line strength in HITRAN database, we make temperature compensation to reduce measurement error of concentration. The experiment proves that, there is a large decrease in variation range of the second harmonic peak on the influence of temperature after temperature compensation. And thus can further realize a higher precision measurement of gas concentration.

\section{References}

[1] J.A Nwaboh, O.Werhahn, P.Ortwein, Laser-spectrometric gas analysis:CO2-TDLAS at 2um, J. Meas. Sci. Technol. 24 (2013) 015202

[2] A. Guha, I. Schoegl, Tomographic TDLAS Using WMS-2fand Tikhonov Regularization,J. Appl. Opt. 53 (2014) 8095

[3] Chang Liu, Lijun $\mathrm{Xu}$, Zhang Cao, Measurement of nonuniform temperature distribution by combining line-of-sight TDLAS with regularization methods, J. Instrumentation. 1 (2014) 43-57

[4] Hua Xia, Fengzhong Dong, Bian Wu, Sensitive absorption measurements of hydrogen sulfide at 1.578 um using wavelength modulation spectroscopy, J. Chin. Phys. B. 24 (2015) 034204

[5] Guangle Zhang, Jianguo Liu, Ruifeng Kan, Simulation studies of multi-line line-of-sight tunable-diode-laser absorption spectroscopy performance in measuring temperature probability distribution function, J. Chin. Phys. B. 23 (2014) 124207 
[6] C. S. Goldenstein, R. M. Spearrin, Wavelength-modulation spectroscopy near 2.5 um for H2O and temperature in high-pressure and -temperature gases, J. Appl. Phys. B. 116(2014) 705-716 